\section{Neuer Wind durch EMAS II?}

\author{
Vor sieben Jahren wurde die EG-Öko-Audit-Verordnung (EMAS) verabschiedet. \\ Wie von vornherein vorgesehen, wurde das neve Instrument in einem Revisi- \\ onsprozess überprüft. Nach jahrelanger Diskussion wurden jetzt die letzten of- \\ fenen Fragen durch den Vermittlungsausschuss von Europäischem Parlament und \\ Rat geklärt. Das Inkrafttreten von EMAS II wird für März/April erwartet. Was \\ sind die wesentlichen Neverungen?
}

\section{W}

Von Jens Pape

Land hat die EMAS-Verordnung in Deutschland Anklang gefunden. Allerdings ist in den vergangenen Monaten eine zunehmende $\mathrm{Zu}$ rückhaltung hinsichtlich der Neu-Eintragungen zu verzeichnen und für viele EMAS-Standorte der ersten Stunde stellt sich die Frage, ob eine Revalidierung durchgeführt werden soll. Die Antwort darauf dürfte genauso wie die Zukunft des ÖkoAudits davon abhängig sein, was die revidierte Fassung der Verordnung mit sich bringt. Bleibt bzw. wird hierdurch EMAS II für die Unternehmen attraktiv?

Bei der Durchsicht der konsolidierten Fassung von EMAS II fällt zunächst auf, dass es gelungen ist, die Verordnung, die mittlerweile ihre Funktionsfähigkeit als innovatives Instrument des betrieblichen Umweltschutzes unter Beweis gestellt hat, praxisgerechter zu gestalten. Beispielsweise ist der für die betriebliche Praxis relevante Part jetzt im Wesentlichen in den klarer strukturierten Anhängen des Verordnungstextes zu finden. Auch in die Begriffsbestimmungen wurde mehr Klarheit gebracht, die sich einerseits in Korrekturmaßnahmen der bereits gebräuchlichen EMAS-Terminologie widerspiegelt: So wird ,environmental performance“ nun mit „Umweltleistung“ übersetzt - um nur ein wichtiges Beispiel zu nennen - und findet damit eine wesentlich glücklichere Übersetzung als dies mit der bisherigen ,kontinuierlichen Verbesserung des betrieblichen Umweltschutzes" geschehen ist. Andererseits ergänzen Begriffe aus der ISO 14.000-Normenfamilie die Terminologie von EMAS II.

Neben diesen strukturellen und terminologischen Verbesserungen sind in EMAS II auch systemprägende Änderungen und Neuerungen hinsichtlich einzelner Systemkomponenten vorgenommen worden. Die wesentlichen Änderungen werden im Folgenden kurz dargestellt.

\section{Umsetzungsleitlinien als Hilfe}

Mit EMAS II werden Leitlinien (,guidelines“) mit unterschiedlicher Verbindlichkeit verabschiedet. Umsetzung und Anwendung der Verordnung in der
Praxis sollen dadurch weiter unterstïtzt werden. Die Themenfelder sowie inhaltliche Schwerpunkte der Leitlinien sind in Abbildung 1 dargestellt.

\section{Öffnung des Adressatenkreises}

Eine wesentliche Änderung in EMAS II ist die vollständige Öffnung des Adressatenkreises. War die Teilnahme bisher auf gewerbliche Unternehmen beschränkt und der Anwendungsbereich lediglich auf nationaler Ebene über (Erweiterungs-) Verordnungen auf weitere Branchen ausgedehnt worden, so ist mit EMAS II jedem die Teilnahme am Gemeinschaftssystem möglich, beispielsweise auch der Bau- und Landwirtschaft.

\section{EMAS II als ISO 14.001 plus}

Im Gegensatz zu EMAS I verzichtet EMAS II auf eigene Vorschriften zur Regelung von Aufbau und Ablauf eines Umweltmanagementsystems und verweist statt dessen in Anhang I A auf den Abschnitt 4 der DIN EN ISO 14.001 Norm und die darin dokumentierten Anforderungen.

Durch diese Einbeziehung der ISO-Norm in EMAS II ist es gelungen, das Verhältnis der beiden Standards zu entspannen, ohne einerseits eines der beiden Systeme auf- oder abzuwerten oder andererseits die anspruchsvolleren Inhalte der EMASVerordnung zu tangieren. Durch diese Integrationslösung werden Unternehmen zunächst nicht gezwungen, sich für eines der Systeme zu entscheiden.

Gleichzeitig entpuppt sich die oftmals beklagte Konkurrenz der beiden Systeme bei genauerer Betrachtung doch eher als komplementäre Beziehung (2): Die ISO 14.001 unterstützt durch ihre Anwender- und Umsetzungsfreundlichkeit die Im-

\begin{tabular}{|c|c|c|c|c|}
\hline \multicolumn{5}{|c|}{ Abb. 1: Leitlinien zu EMAS II } \\
\hline Guideline zu & Titel & Thema & Ermächtigung in EMAS II & Status der Leitlinie \\
\hline \multicolumn{2}{|c|}{ Umwelterklärung } & "Guidance on the & Erläuterungen & zu Inhalt und \\
\hline \multirow[t]{2}{*}{ Anh. III 3.1} & erläuternd & & & \\
\hline & \multicolumn{2}{|c|}{ environmental statement" } & \multirow{2}{*}{\multicolumn{2}{|c|}{$\begin{array}{l}\text { Veröffentlichung der Umwelterklę } \\
\text { u.a.Bedingungenzurausnahmsweisen }\end{array}$}} \\
\hline \multicolumn{2}{|c|}{ Begutachtungs- und } & „'Guidance on Verification and & & \\
\hline Anh. III 3.4 & verbindlich & \multirow{3}{*}{ Validation Frequency" } & & \\
\hline \multicolumn{2}{|c|}{ Validierungshäufigkeit } & & \multicolumn{2}{|c|}{ Abweichung vom einjährigen Validie- } \\
\hline rungszyklus & Anh. V 5.6 & & & \\
\hline \multicolumn{3}{|c|}{ Umweltaspekfe „Guidance on the Id entification } & \multicolumn{2}{|c|}{ Erhebung von signifikanten Umwelta- } \\
\hline spekten & - & erläuternd & & \\
\hline (Anh. VI) & \multicolumn{2}{|c|}{ of Environmental Aspects and } & \multicolumn{2}{|c|}{ undAnleitungfür dieSignifikanzbewer- } \\
\hline \multicolumn{5}{|l|}{ fung } \\
\hline & \multirow{2}{*}{\multicolumn{2}{|c|}{$\begin{array}{l}\text { Assessment of their Significance" } \\
\text { "Guidance on the entity to be }\end{array}$}} & & \\
\hline Standort / & & & \multicolumn{2}{|c|}{ u.a.AusnahmeregelungenzumStandort } \\
\hline Art. 2s) & \multicolumn{4}{|l|}{ verbindlich } \\
\hline
\end{tabular}


plementierung eines Umweltmanagementsystems und damit die Verbreitung betrieblicher Umweltmanagementsysteme. Mit der weitergehenden Umsetzung der Verordnung - insbesondere durch die Pflicht zur Erstellung einer Umwelterklärung, die Standortregistrierung und die Überprüfung durch einen akkreditierten Umweltgutachter wird einem Ziel mit starkem umweltpolitischen Charakter Rechnung getragen. Unterstrichen wird durch die Einbeziehung der ISO 14.001 in EMAS II somit auch, dass die Verordnung auf eine StarPerformance ausgelegt ist. Dies kommt in den weiter bestehenden Zusatzanforderungen hinsichtlich

der Gesetzeseinhaltung,

- einer quantifizierbaren und messbaren Verbesserung der Umweltleistung in stofflicher und energetischer Hinsicht,

der aktiven Einbeziehung der Arbeitnehmer,

der externen Begutachtung

sowie durch das Bekenntnis zu einer aktiven externen Kommunikation unter anderem anhand der Umwelterklärung zum Ausdruck.

\section{- Organisation statt Standort}

Vor dem Hintergrund der Erweiterung des Adressatenkreises und der Einbeziehung der ISO 14.001 wurde die Ablösung des Standortbegriffs und dessen Ersatz durch den Organisationsbegriff notwendig. Gleichzeitig bleibt aber der Standort - im Gegensatz zur ISO-Norm - die kleinste validierungsfähige Einheit. Auch eine grenzüberschreitende Zusammenfassung von Standorten zu einer Organisation ist nicht möglich. Ein weiterer Punkt, der die weiterhin gültige Bedeutung des Standortbezugs der EMAS-Verordnung unterstreicht, ist die oben angesprochene „Performanceorientierung", also die kontinuierliche Verbesserung der managementorientierten und operativen Umweltleistung des Unternehmens, die weiterhin standortbezogen nachzuweisen ist (,local accountability“).

Insbesondere für Unternehmen mit Filialen etwa aus der Dienstleistungsbranche oder dem Handel wird es durch die Einführung des Organisationsbegriffs dennoch erleichtert, Standorte zu einer validierungsfähigen Organisation zu verbinden.

\section{- Einbezug indirekter Umweltwirkungen}

Die Identifizierung signifikanter Umweltaspekte, auf die das Umweltmanagement ausgerichtet werden soll, war und ist ein wesentliches EMAS-Element. Mit der Erweiterung des Anwendungsberei- ches und dessen Ausdehnung insbesondere auch auf den Dienstleistungssektor ist die Übernahme von indirekten Umweltaspekten in das Anforderungsprofil der Verordnung notwendig geworden. In Abhängigkeit des Zentralgeschäftes der Organisation rückt so neben organisatorischen Aspekten und Fragen der Betriebsökologie auch verstärkt die Produktökologie ins Zentrum der Betrachtung. Nicht zuletzt aufgrund der Komplexität dieses Themas wird daher zusammen mit EMAS II für die Erhebung von signifikanten Umweltaspekten sowie zur Signifikanzbewertung eine erläuternde Leitlinie veröffentlicht werden (vgl. Abb. 1).

\section{- Neues EMAS-Zeichen}

Mit Inkrafttreten von EMAS II stehen für die EMAS-Teilnehmer zwei Versionen des neuen EMAS-Zeichens zur Verfügung (Abb. 2). Neben den entsprechenden Passagen in EMAS (vgl. Art.

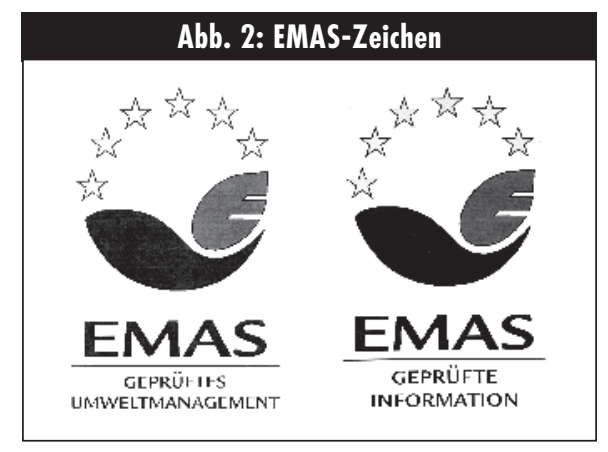

8 und Anh. IV EMAS II) werden die Bedingungen hinsichtlich der Verwendung des EMAS-Zeichens in einer entsprechenden Leitlinie (vgl. Abb.1) geregelt.

Mit dem neuen Logo soll EMAS als Markenzeichen für eine umweltgerechte Produktion oder Dienstleistung in der Öffentlichkeit etabliert werden. Das EMAS-Logo soll auf die Beteiligung der Organisation an EMAS hinweisen.

Das Logo ,geprüfte Information“ soll Informationen, die in direktem oder indirektem Zusammenhang mit Produkten, Tätigkeiten oder Dienstleistungen stehen, Glaubhaftigkeit verleihen, darf aber nur verwendet werden, wenn diese Informationen ,,von einem Umweltgutachter als korrekt und nicht irreführend, begründet und nachprïfbar, relevant und im richtigen Kontext verwendet, repräsentativ für die Umweltleistung der Organisation insgesamt, unmissverständlich und wesentlich in Bezug auf die gesamten Umweltauswirkungen“ bestätigt werden (3) . „Geprüfte Informationen“ stammen somit vor allem aus der Umwelterklärung.
Die Verwendung der EMAS-Zeichen auf Produkten und ihren Verpackungen ist (weiterhin) genauso untersagt, wie ihre Verwendung im Rahmen von Vergleichen mit anderen Produkten, Tätigkeiten und Dienstleistungen.

\section{Häufigkeit der Validierung}

Die inhaltlichen Anforderungen der Umwelterklärung wurden durch den Anhang III konkretisiert. EMAS II sieht eine jährliche Aktualisierung sowie Validierung der Umwelterklärung vor. Für Unternehmen mit bis zu 50 Mitarbeitern soll eine Ausnahme von der jährlichen Aktualisierungspflicht durch eine EU-Leitlinie bestehen.

Der Validierungszyklus, das heißt der Zeitraum in dem alle Umweltaspekte überprüft und begutachtet sein müssen, und damit eine neue, konsolidierte Fassung der Umwelterklärung, ist weiterhin auf drei Jahre angelegt.

\section{"Wir setzen ein Zeichen"}

Mit dem Inkrafttreten von EMAS II tritt EMAS I außer Kraft. Bisher registrierte Standorte gehen in das neue EMAS II Register über. Bei der nächsten Revalidierung wird dann hinsichtlich der Anforderungen nach EMAS II geprüft werden.

Unter dem Slogan „Wir setzen ein Zeichen“ steht eine zeitgleich mit dem Inkrafttreten von EMAS II gestartete Gemeinschaftsinitiative von Bund, Ländern, der deutschen Wirtschaft, der Gewerkschaften und der Umweltverbände zur Erhöhung des Bekanntheitsgrades der Verordnung und insbesondere des neuen EMAS-Logos (3). Initiator und Promotor der Kampagne ist der Umweltgutachterausschuss.

\section{Anmerkungen}

(1) Hansen, M.: Alles neu durch EMAS II? In: FORUM Umweltmanagement, Vol. 1, Ausgabe 2, Juli 2000, S. 10-14. (2) Wassmuth, B.: EMAS 2 - Fortschritt oder Rückschritt? In: BfuP, 5/1999, S. 543-553.

(3) Vgl. www.emas-logo.de

\section{Der Autor}

Jens Pape ist wissenschaftlicher Mitarbeiter an der Universität Hohenheim sowie Mitglied im Umweltgutachterausschuss und im Doktoranden-Netzwerk ÖkoAudit e.V.

Kontakt: Universität Hohenheim, Institut (410A), Fachgebiet Agrarinformatik und Unternehmensführung, 70593 Stuttgart. Tel. 0711/ 459-2574, Fax -3481,E-Mail: pape@uni-hohenheim.de 
(c) 20I0 Authors; licensee IÖW and oekom verlag. This is an article distributed under the terms of the Creative Commons Attribution Non-Commercial No Derivates License (http://creativecommons.org/licenses/by-nc-nd/3.o/), which permits unrestricted use, distribution, and reproduction in any medium, provided the original work is properly cited. 